\title{
Retour sur l'origine du vaccin antivariolique
}

\author{
Robert Drillien ${ }^{1}$ \\ Daniel Garin ${ }^{2}$ \\ ${ }^{1}$ IGBMC, 1, rue Laurent-Fries \\ 67404 Illkirch-Graffenstaden, France \\ $<$ drillien@igbmc.fr> \\ 2 MT2i, 15, rue des Bergeronnettes, \\ CS52613,
}

38036 Grenoble cedex 2, France

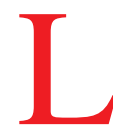

e vaccin antivariolique est à l'origine d'un certain nombre de contrevérités. Dans une émission radiophonique récente sur une radio nationale de grande écoute française, une adepte des théories «complotistes » affirmait que des rapports de l'OMS démontraient clairement que l'éradication de la variole n'était pas due au vaccin. C'est une désinformation absolument scandaleuse. Le succès du vaccin antivariolique a été exceptionnel et la variole humaine, « le plus grand fléau qu'ait connu l'humanité depuis des millénaires » selon Jenner, a pu être entièrement éradiquée grâce à son utilisation systématique. Beaucoup pensent aussi que ce vaccin est constitué par le virus cowpox. C'est inexact. Si ce virus a probablement été le premier utilisé par Jenner, le principe actif du vaccin est constitué d'un autre virus, le virus de la vaccine. C'est sans conteste le premier vaccin des temps modernes qui a d'ailleurs donné son nom à la procédure même de « vaccination ». L'histoire de ce vaccin a été rapportée à de multiples reprises au cours des générations successives, depuis son introduction à la fin du XVIII ${ }^{\mathrm{e}}$ siècle. Mais cette histoire s'enrichit régulièrement de nouveaux éclairages grâce notamment à la découverte d'anciens échantillons de vaccins (provenant de musées ou de collections privées) et à leur séquençage génomique. L'introduction de la protection antivariolique s'est inspirée de pratiques très anciennes en cours dans l'Orient et importées en Europe au début du XVIII ${ }^{\mathrm{e}}$ siècle. À cette époque, on cherchait à se protéger contre la variole par incision superficielle de la peau et inoculation sur la plaie avec de la lymphe prélevée de pustules de personnes atteintes d'une variole naturelle. La pratique de l'inoculation, désignée par la suite sous le nom de « variolisation », n'entraînait la plupart du temps que des lésions cutanées relativement restreintes au site inoculé, une fièvre et un affaiblissement général, un ensemble de symptômes bien moins graves que la variole acquise naturellement via les voies respiratoires. L'inoculation par voie cutanée permet au système immunitaire de se mettre en place, évitant ainsi l'apparition de la variole «maladie ». Même si la « variolisation » entraînait de temps en temps une variole mortelle et, à l'occasion, était la source d'une nouvelle épidémie, le rapport bénéfice/risque fut reconnu comme favorable, si bien que la méthode se répandit largement. Dans ce contexte, plusieurs personnes pratiquant la variolisation, dont le médecin anglais Edward Jenner (1749-1823), remarquèrent que les sujets exposés à des infections antérieures avec le cowpox (poxvirose bovine dont l'agent responsable est distinct du virus de la vaccine), contracté au contact de vaches laitières atteintes aux pis, ne montraient aucun symptôme lors de la variolisation et restaient indemnes lors d'une épidémie naturelle. Les pustules sur la main des personnes atteintes de cowpox rappelaient l'aspect des pustules des sujets malades de variole d'où le terme « variole de la vache » pour désigner le cowpox. Le pas décisif que fit Jenner (et plusieurs de ses contemporains oubliés de l'histoire) a consisté à inoculer la lymphe provenant de pustules d'une fermière atteinte de cowpox à un enfant, puis d'éprouver cet enfant par une variolisation, méthode répandue à cette époque. L'enfant n'ayant montré aucun des signes infectieux caractéristiques de la variolisation, Jenner a poursuivi sa méthode pour finalement la publier [1] et la promouvoir en Angleterre et à l'étranger. Il démontra par ailleurs que le cowpox pouvait être transmis par passage en cascade de personne à personne, sans perdre son pouvoir protecteur contre la variolisation ou la variole lorsqu'une épidémie sévissait. 
Les convictions de Jenner quant à l'origine du cowpox n'ont cessé d'intriguer la communauté scientifique. Jenner avait constaté les lésions de cowpox sur les pis des vaches et leur transmission aux personnes chargées de la traite, mais aussi des lésions sur les sabots de chevaux. Selon lui, ces lésions chez le cheval devaient être la source de l'infection des vaches par l'intermédiaire des personnes en contact avec ces deux espèces domestiques. Par ailleurs, il était de notoriété publique que les maréchaux-ferrants ne présentaient pas de lésions après variolisation, suggérant qu'ils étaient protégés après contact avec un cheval souffrant de poxvirose équine (horsepox). Jenner et ses contemporains ont ainsi testé le recours à des prélèvements de pustules de poxvirose équine pour réaliser des « vaccinations » elles aussi couronnées de succès.

La notion qu'il existe une espèce de poxvirus unique capable d'infecter indifféremment la vache ou le cheval est tout à fait conforme aux connaissances actuelles, car ces deux animaux sont susceptibles d'être infectés par un poxvirus de rongeur, animal considéré comme le véritable réservoir du cowpox et de la poxvirose équine [2]. En effet, le virus de l'espèce cowpox peut infecter de nombreux animaux dont le chat qui est un vecteur de transmission à l'homme aujourd'hui [2]. Le cowpox de la vache et la poxvirose équine ont persisté pendant une bonne partie du XVIII ${ }^{\mathrm{e}}$ siècle pour disparaitre totalement aujourd'hui peut-être en raison des améliorations dans l'hygiène des élevages. Avec la propagation d'une nouvelle procédure de prévention de la variole à travers l'Europe et les autres continents, de nombreux prélèvements de lésions provoquées par le cowpox ou le horsepox ont été effectués à des fins de vaccination humaine. Initialement, ces vaccins étaient entretenus par passage de bras en bras (propageant par la même occasion d'autres infections telles que la syphilis). Cette méthode fut progressivement remplacée par le passage du vaccin sur la peau d'animaux (vache, mouton, lapin). Certains d'ailleurs mélangeaient des lymphes provenant de plusieurs animaux ou réalisaient une vaccination et une variolisation simultanée [3]. Ainsi, trois facteurs principaux pourraient expliquer la diversité des vaccins antivarioliques qui ont été transmis jusqu'à nos jours :

- la multiplicité des sources animales des vaccins employés ;

- le fait de mélanger des virus de provenance distincte facilitant ainsi la recombinaison entre génomes viraux voisins ; - les nombreux passages chez l'homme et chez l'animal.

Si Jenner utilise le mot «virus » dans ses écrits, il a fallu attendre la naissance de la virologie et son essor au début $\mathrm{du} \mathrm{xx}^{\mathrm{e}}$ siècle pour saisir l'originalité de ces agents infectieux. Au fur et à mesure des avancées de cette discipline, on s'est rendu compte que les vaccins antivarioliques d'origines diverses présentaient des phénotypes distincts avec une diversité de propriétés : notamment la capacité de culture chez l'animal et l'importance de la réaction inflammatoire observée au site d'inoculation. Le nombre d'accidents de vaccination variait au gré des souches utilisées. Mais c'est surtout le séquençage des génomes viraux de ces dernières années qui a permis de mieux comprendre ces différences. Une variété importante de vaccins antivarioliques a été utilisée dans le passé et beaucoup d'entre eux sont sans doute perdus pour toujours même si un regain d'intérêt pour ce sujet amène à rechercher des vaccins anciens dans les musées ou les collections privées. À l'heure actuelle, la séquence complète du génome d'une quinzaine de souches du virus de la vaccine est disponible dans les bases de données publiques (tableau 1). Ces génomes, bien que suffisamment proches pour constituer une quasi-espèce virale, restent distincts les uns des autres. La partie centrale du génome, recouvrant environ $150 \mathrm{kbp}$, renferme les gènes essentiels à la multiplication virale, avec une variabilité nucléotidique limitée. Les différences génomiques majeures se situent dans les répétitions terminales inversées et les régions adjacentes (environ $20 \mathrm{kbp}$ à chaque extrémité) qui codent des fonctions non essentielles ou « accessoires » pour la multiplication virale en culture cellulaire, mais importantes pour les interactions avec l'organisme hôte et la spécificité d'espèce. Il s'agit notamment de protéines modulatrices de la réponse immunitaire innée (inhibiteurs de cytokines, des interférons, de l'apoptose). Une grande variabilité de séquences est observée dans ces régions avec des délétions et des mutations ponctuelles inactivant certains gènes selon la souche examinée : remarquablement, aucune souche de virus de la vaccine ne possède une version intacte de la totalité de ces gènes « accessoires ». À partir de ces observations, les différentes souches du virus de la vaccine ont été classées en trois groupes (figure 1):

- les souches eurasiennes;

- les souches d'Amérique du Nord ;

- les souches d'Amérique du Sud [4, 5].

Toutes proviennent de souches isolées en Europe à la fin du $\mathrm{XVII}^{\mathrm{e}}$ et au début du XIX ${ }^{\mathrm{e}}$ siècle. Certains auteurs ont émis l'hypothèse que toutes les souches ont un parent commun, un poxvirus équin : Louis Pasteur se serait donc alors fourvoyé en utilisant le terme de vaccination en référence à la souche d'origine bovine utilisée par Jenner et nous devrions alors utiliser le terme «d'équination». Mais les modalités variées de prélèvement dans la nature et les mélanges réalisés paraissent peu compatibles avec cette source unique de virus. Dans la plupart des souches examinées l'ADN d'un seul clone viral a été séquencé. Lorsque l'ADN de plusieurs clones d'une même souche a été séquencé, il y a toujours une hétérogénéité significative reflétant la dérive génétique qui se produit au cours des passages successifs. 
Tableau 1 Souches du virus de la vaccine séquencées à ce joura ${ }^{\text {. }}$

\begin{tabular}{|c|c|c|c|c|}
\hline Souche virale & $\begin{array}{l}\text { Taille du génome } \\
\text { (nucléotides) }\end{array}$ & Pays d'origine & $\begin{array}{l}\text { Propriété } \\
\text { remarquable }\end{array}$ & Commentaire \\
\hline Lister-107 & 189421 & France & $\begin{array}{l}\text { Vaccin (produit } \\
\text { initialement à l'institut } \\
\text { Lister (Angleterre) }\end{array}$ & $\begin{array}{l}\text { D'autres séquences de souches } \\
\text { Lister sont également disponibles } \\
\text { dont VACV-LO, LC16m8, LC16m0, } \\
\text { L-IVP, WAU86/88-1, Butantan }\end{array}$ \\
\hline Copenhague & 191737 & Danemark & Vaccin & $1^{\text {re }}$ souche séquencée en 1990 \\
\hline CVA & 192353 & Turquie & Vaccin & Souche parentale de la souche MVA \\
\hline MVA & 177923 & Allemagne & $\begin{array}{l}\text { Vaccin non réplicatif } \\
\text { en cellules humaines }\end{array}$ & $\begin{array}{l}\text { Très nombreuses délétions } \\
\text { et mutations après } 596 \text { passages } \\
\text { en cellules d'embryon de poulet, } \\
3 \text { séquences disponibles }\end{array}$ \\
\hline Tashkent & 182481 & Ouzbékistan & Vaccin & $\begin{array}{l}\text { Souche virulente dont l'utilisation } \\
\text { en vaccination a été abandonnée }\end{array}$ \\
\hline TianTan & 196263 & Chine & Vaccin & Plusieurs clones séquencés \\
\hline IHD-W & 195821 & États-Unis & Vaccin & $\begin{array}{l}\text { Dérivé de virus IHD-J (souche } \\
\text { japonaise du virus IHD) }\end{array}$ \\
\hline Dryvax & 196939 & États-Unis & Vaccin & $\begin{array}{l}\text { Nombreux clones séquencés } \\
\text { dont la souche Acambis } 2000\end{array}$ \\
\hline WR & 194711 & États-Unis & Souche de laboratoire & Souche neurovirulente chez la souris \\
\hline Duke & 199960 & États-Unis & Patient & $\begin{array}{l}\text { Souche isolée d'un patient } \\
\text { avec complications vaccinales }\end{array}$ \\
\hline IOC & 192017 & Brésil & Vaccin & 2 clones séquencés \\
\hline Cantagalo & 181774 & Brésil & Isolat naturel & Isolée de vaches malades \\
\hline Serro-2 & 184572 & Brésil & Isolat naturel & Isolée d'un trayeur âgé de 30 ans \\
\hline Horsepox & 212633 & Mongolie & Isolat naturel & Isolée d'un cheval malade \\
\hline Mulford 1902 & 196464 & États-Unis & Vaccin & $\begin{array}{l}\text { Souche vaccinale la plus ancienne } \\
\text { connue (1902) }\end{array}$ \\
\hline Rabbitpox virus & 197731 & États-Unis & $\begin{array}{l}\text { Apparu dans } \\
\text { l'élevage des lapins }\end{array}$ & $\begin{array}{l}\text { Pathogène pour le lapin (origine } \\
\text { inconnue) }\end{array}$ \\
\hline
\end{tabular}

a Lorsque plusieurs génomes ont été séquencés, la taille du génome le plus grand est fournie. Les séquences ne comprennent pas toujours les petites répétitions en tandem que l'on retrouve aux extrémités ( $\approx 6 \mathrm{kbp}$ de part et d'autre du génome).

Quoiqu'il en soit, l'ensemble de ces vaccins appartient clairement à la quasi-espèce virale "virus de la vaccine ", suffisamment éloignée des souches de virus cowpox caractérisées à ce jour. Si le virus cowpox est bien apparenté au virus de la vaccine par son appartenance au genre Orthopoxvirus, il possède le plus grand des génomes des virus de ce genre. Son génome renferme l'éventail de gènes le plus important avec les gènes dits « accessoires » intacts. On en déduit que le cowpox est le virus le plus proche de l'ancêtre commun à l'ensemble des Orthopoxvirus et que la spécificité à l'espèce humaine caractéristique du virus de la variole a été acquise simultanément avec une réduction de la taille du génome.

Les analyses génétiques les plus récentes placent le poxvirus équin au sein de l'espèce « virus de la vaccine » et amènent à penser qu'un virus équin serait à l'origine de nos souches actuelles de virus vaccinal, hypothèse déjà formulée par Jenner. Cette idée a été davantage étayée récemment par la découverte et le séquençage d'une ancienne ampoule de vaccin (provenant de la collection privée de José Esparza) dont le génome renfermait des séquences propres au poxvirus équin [6]. L'hypothèse s'appuie cependant sur la séquence d'une seule souche de virus équin isolé en Mongolie en 1976 (séquence publiée en 2006) et sa proximité génétique avec des virus équins circulant il y a deux siècles en Europe est incertaine. Aujourd'hui, les poxvirus équins ne circulent plus dans la nature. Comme autre explication, le virus équin provenant de Mongolie dériverait d'un virus vaccinal transmis aux chevaux par des animaux ou personnes vaccinés à une époque où la vaccination 


\section{éditorial}

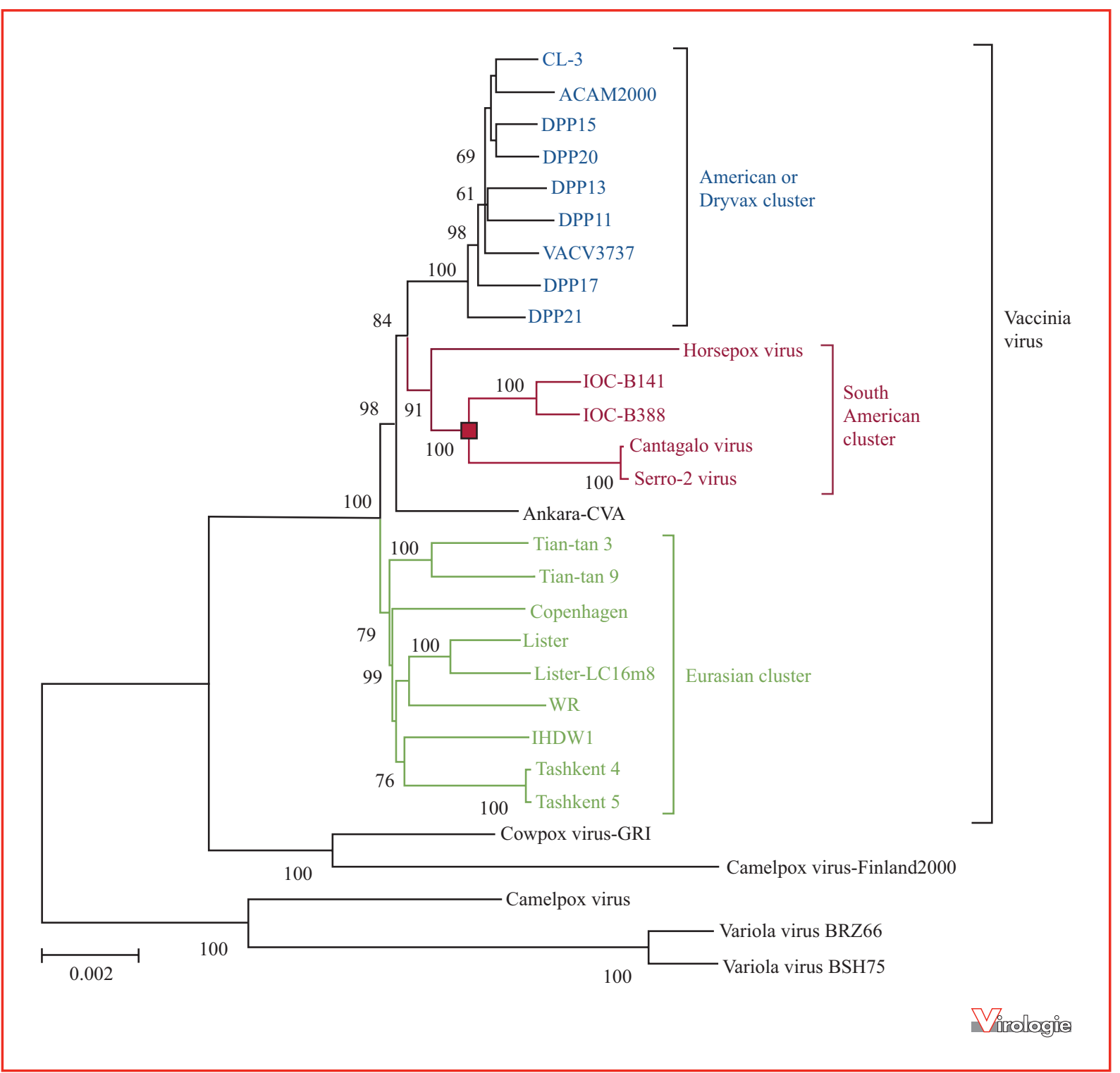

Figure 1. Phylogénie des souches du virus de la vaccine séquencées à ce jour (reproduite depuis la référence [5], avec l'aimable autorisation des éditions Elsevier).

* Les régions génomiques les mieux conservées (environ 95000 nucléotides) ont été alignées et le nombre de substitutions par site (barre d'échelle en bas à gauche) a été utilisé pour déterminer la proximité entre chaque souche virale (Kimura 2-parameter substitution model avec 100 replicas bootstrap). Notons également la représentation des distances phylogéniques des virus cowpox, camelpox et variole.

était une pratique peu rigoureuse. Ce phénomène ne serait pas unique puisque de tels événements ont bien eu lieu au Brésil et en Inde où tout porte à croire que des virus retrouvés chez les bovins ou buffles respectivement sont bien des virus de la vaccine authentiques, génétiquement proches des souches vaccinales [7]. Elles sont d'ailleurs source d'infections humaines et de dissémination à d'autres troupeaux. Le virus de la vaccine a été isolé à plusieurs reprises chez des rongeurs au Brésil et cela est fortement évocateur de virus de vaccins retournés à l'état sauvage. Si le vaccin antivariolique devait être utilisé à nouveau en raison d'une résurgence de la variole, la plupart des pays auraient recours aux principaux vaccins conseillés par l'OMS durant les campagnes de vaccination intensive. Ces vaccins vivants dits de première génération (produits chez l'animal) ou de deuxième génération (souches 
identiques à celles de première génération mais produites en culture cellulaire) et inoculés par voie cutanée sont source d'accidents vaccinaux conséquents (vaccine généralisée, encéphalite, myopéricardite, eczéma vaccinatum). D'autres vaccins atténués de manière empirique (MVA, LC16m8) (troisième génération), et qui ont fait l'objet de nombreux essais chez des volontaires, pourraient être employés chez des sujets susceptibles de faire des réactions indésirables au vaccin traditionnel (personnes immunodéprimées, souffrant d'eczéma ou femmes enceintes). Mais le développement industriel de ces vaccins pourtant disponibles est malheureusement fortement compromis par l'absence de demande. En effet, peu de nations ont réalisé des commandes pour protéger leurs populations devant un risque de variole considéré comme faible ou impossible à évaluer.

Depuis l'abandon de la vaccination antivariolique, le virus de la vaccine a été utilisé en tant que vecteur viral avec de nombreuses applications en recherche fondamentale, en vaccination et, plus récemment, en thérapie anticancéreuse. La connaissance approfondie du génome des différentes souches, révélée par l'étude des vaccins historiques, peut guider la réalisation de nouvelles stratégies biotechnologies basées sur ce virus. La création d'un virus de la vaccine à partir d'ADN synthétique rapportée en janvier 2018 [8] ouvre de nouvelles perspectives d'application mais suscite également des inquiétudes quant à une utilisation malveillante de cette technologie.
Liens d'intérêts: R. Drillien déclare être membre du comité d'experts de l'OMS, Advisory comittee on variola virus research. Les autres auteurs déclarent ne pas avoir de liens d'intérêts en rapport avec cet article.

\section{Références}

1. Jenner E. An inquiry into the causes and effects of the variolae vaccinae, a disease discovered in some of the western countries of England, particularly Gloucestershire and known by the name of the cow-pox. London: Sampson Low, 1798.

2. Essbauer S, Pfeffer M, Meyer H. Zoonotic poxviruses. Vet Microbiol $2010 ; 140: 229-36$.

3. Esparza J, Schrick L, Damaso CR, Nitsche A. Equination (inoculation of horsepox): an early alternative to vaccination (inoculation of cowpox) and the possible role of horsepox virus in the origin of the smallpox vaccine. Vaccine 2017 ; 35 : 7222-30.

4. Qin L, Favis N, Famulski J, Evans DH. Evolution of and evolutionary relationships between extant vaccinia virus strains. J Virol $2015 ; 89$ : 1809-24.

5. Damaso CR. Revisiting Jenner's mysteries, the role of the Beaugency lymph in the evolutionary path of ancient smallpox vaccines. Lancet Infect Dis 2018; 18 : e55-e63.

6. Schrick L, Tausche SH, Wojciech Dabrowski P, Damaso C, Esparza J, Nitsche A. An early American smallpox vaccine based on horsepox. N Engl J Med 2017; 377 : 1491-2.

7. Oliveira JS, Figueiredo PO, Costa GB, et al. Vaccinia virus natural infections in Brazil: the good, the bad, and the ugly. Viruses 2017; 9, pii : E340.

8. Noyce RS, Lederman S, Evans DH. Construction of an infectious horsepox virus vaccine from chemically synthesized DNA fragments. PLoS One 2018 ; 13 : $\mathrm{e} 0188453$. 